International Journal of Research in Engineering and Innovation
(IJREI)
jJREI

\title{
Computational analysis of sloshing in 3-D rectangular tank under seismic excitation with and without baffle
}

\author{
Puneet Kumar Nema, Kapil
}

Department of Mechanical Engineering, Madhav Institute of Technology and Science Gwalior

\begin{abstract}
In this research analysis, liquid sloshing phenomenon in a rectangular tank was simulated using ANSYS-FLUENT, subjected to various Seismic Excitation frequency at different water fill levels, with and without baffle. Two different water fill levels of $60 \%$ and $80 \%$ are used. For Analysis of 3-D incompressible two- phase flow in a partially filled water tank the volume of fluid (VOF) method based on the finite volume method (FVM) has been used. User Defined Function (UDF) in cell zone condition is used for generation of External Seismic excitation. Variable time method is adopted for simulation of tank for $20 \mathrm{sec}$. The investigation shows that the sloshing effect will become extreme, if the tank is subjected to resonant excitation Frequencies, and wall forces will be intensified. The time variation of pressure, and the free surface movement of liquid is been presented. By using baffle of certain height (critical height), the liquid does not reach at top of tank and when height of baffle is equal to water level, the free water surface represent linear behavior in each section.

(C) 2019 ijrei.com. All rights reserved
\end{abstract}

Keywords: Liquid Sloshing; Seismic Excitation; Computational Fluid Dynamics; VOF method, Finite Volume Method.

\section{Introduction}

Sloshing phenomenon can be explained as any action of the free liquid surface inside any container. The free liquid surface movement can be caused by disturbance to partially filled liquid tanks [1]. For sloshing effect to occur inside any tank, the liquid must have a free motion to constitute a slosh dynamic problem, where the motion of liquid can interrelate with container walls to alter the system dynamic stability. The movement of liquid with sloshing effect is of prime concern in several engineering applications such as in spacecraft rockets, in marine ships and vehicles transporting liquid (for example oil, water, LNG and gasoline), oscillation in a reservoir due to seismic waves, sloshing in Nuclear Reactors and several others. [2]

Hydrodynamic load exerted by liquid in sloshing phenomenon can cause severe structural damage. One of the major solutions to avoid the violent free surface oscillation is installing baffles inside the liquid tanks [1]. The sloshing primarily depends on the excitation type and its frequency, storage tank geometry, motion of liquid. The tank excitation can be of any type, i.e. periodic, spontaneous, and sinusoidal and its effect on the tank could be planar, non-planar, lateral, rotational, irregular beating, parametric, symmetric or asymmetric. [3]

Interest in sloshing phenomenon has grown and been investigated for past few decades. Numerous studies have been performed using theoretical, experimental, and numeric techniques. Initial studies on sloshing [29, 30] were based on Theoretical models. Several Research studies concentrated on linear solutions of sloshing based on potential flow assumptions $[6,7]$. These techniques were accurate but do not give good results on wave overturning, breaking and in case of vicious liquid sloshing. Akyildiz and Unal (2005) conducted an experimental study on pressure variations in both baffled and unbaffled rectangular tanks [26].

Thus to conquer these problems numerical simulation and analysis have become an alternative approach. Numerical studies mostly employed grid-based finite difference method (FDM) [22, 23] and finite element method (FEM) [24-25]. Some comprehensive review on this topic can be found in research literature [5, 19, 20-21]. In recent times, the Computational Fluid Dynamics (CFD) research is playing very significant role to evaluate the liquid sloshing problem. It facilitate time saving and 
cost by eliminating the experimental modeling technique. The CFD modeling approaches are also able to overcome flow limitations experienced in theoretical studies. A lot of work has been done in the application of CFD to liquid sloshing.

Ling Hou et al., (2012); J.H. Jung et al., (2012); V. Singhal et al., (2014); T. Kandasamy et al., (2010) uses VOF (Volume of Fluid) method to track the free surface of sloshing [8-11]. M. Eswaran et al., (2009) [14] studied the sloshing waves using finite element scheme in baffled and without baffled cubic tank with ArbitraryLagrangian-Eulerian (ALE) formulation which adopts the displacement of solid, the pressure and displacement in the fluid as variables to model the coupled system. Cho and Lee (2004) [27] and Cho et al. (2005) [28] simulated the liquid sloshing in two- dimensional (2D) rectangular tank with baffle under horizontal external excitation. The relative baffle heights and its effect on sloshing impact were investigated.

Installation of baffles, either horizontal or vertical inside tank can effectively suppress the sloshing effect and damage caused by it. [31-33].

In this work, the CFD method with Finite Volume Approach is used to investigate the liquid sloshing behaviour in a 3-D rectangular baffled tank which is subjected to seismic excitation of different frequencies. Simulation has been done on ANSYSFluent. Comparative study of sloshing effect by installing baffle has also presented. Seismic excitation is imposed on the tank due to earthquake ground motion. These excitations are employed by Momentum Source input using User defined Function. The volume of fluid (VOF) method is used to track the free surface of sloshing. The idea of the current study is to observe computationally how the baffle height relative to the initial liquid fill levels influences the sloshing phenomenon when the vertical baffle is positioned at the center of the bottom wall of the tank which is externally excited with frequency equal to the natural frequency of the liquid inside the tank.

\section{Mathematical Model}

The sloshing behavior in a liquid tank which can be represented by an incompressible viscous fluid flow with a free surface is governed by the Navier-Stokes equation and the continuity equation.

For 3-dimensional continuity equation and Navier-stokes equation for unsteady flow is as follow:

$$
\begin{aligned}
& \frac{\partial \rho}{\partial t}+\frac{\partial(\rho u)}{\partial x}+\frac{\partial(\rho v)}{\partial y}+\frac{\partial(\rho w)}{\partial z}=\mathbf{O} \\
& \frac{\partial \rho}{\partial t}+\frac{\partial(\rho u)}{\partial \boldsymbol{x}}+\frac{\partial(\rho v)}{\partial y}+\frac{\partial(\rho w)}{\partial z}=\mathbf{O} \\
& \frac{\partial \rho}{\partial t}+\frac{\partial(\rho u)}{\partial x}+\frac{\partial(\rho v)}{\partial y}+\frac{\partial(\rho w)}{\partial z}=\mathbf{O} \\
& \frac{\partial(\rho u)}{\partial t}+\rho\left(u \frac{\partial u}{\partial x}+v \frac{\partial u}{\partial y}+w \frac{\partial u}{\partial z}\right)=\rho x-\frac{\partial p}{\partial x}+\frac{1}{3} \mu \frac{\partial}{\partial x}\left(\frac{\partial u}{\partial x}+\frac{\partial v}{\partial y}+\frac{\partial w}{\partial z}\right)+\mu \nabla^{2} u
\end{aligned}
$$

Where ' $\rho$ ' is the density, ' $t$ ' is time, ' $p$ ' is pressure, $\mu$ is dynamic viscosity, and $u, v, w$ are velocity components in $x, y, z$ direction.

In computational fluid dynamics, the volume of fluid (VOF) method is a technique used for tracking the free surface (or fluidfluid interface) between the phases. In VOF model, the volume fraction of each of the fluids in each computational cell is tracked throughout the domain. This model solves total continuity equation and the result is pressure and volume fraction which shows where the interface is.

The momentum equation is dependent on the volume fractions of all phases through the properties $\rho$ and $\mu$.

$\frac{\partial}{\partial t}(\rho \vec{V})+\nabla \cdot(\rho \vec{V} \vec{V})=-\nabla p+\nabla \cdot(\vec{\tau})+\rho \vec{g}+\vec{F}$

The VOF method uses a characteristic function $\alpha$ to capture the fluid volume and identify the free surface position.

If $\alpha_{b}$ represent the $b^{\text {th }}$ fluid's volume fraction in the cell then the following three conditions are possible:

- $\quad \alpha_{b}=0$, shows the cell is empty (no fluid of $b$ type is present)

- $\alpha_{b}=1$, shows the cell is full (only b type fluid is present).

- $0<\alpha_{b}<1$, shows the cell contain the interface between the $\mathrm{b}^{\text {th }}$ fluid and one or more other type of fluid.

The following equation is used to track the free surface and interface between the phases. For $b^{\text {th }}$ phase, the equation will be:

$$
\frac{1}{\rho_{b}}\left[\frac{\partial}{\partial t}\left(\alpha_{b} \rho_{b}\right)+\nabla \cdot\left(\alpha_{b} \rho_{b} \overrightarrow{V_{b}}\right)=s_{\alpha_{b}}+\sum\left(\dot{m}_{a b}-\dot{m}_{b a}\right)\right]
$$

Where $m_{a b}$ is mass transfer from a to b phase and $m_{b a}$ is mass transfer from $b$ to a phase. $s_{\alpha b}$ shows the source term which permits the use of cavitation model.

For n phases,

$$
\sum_{b=1}^{n} \alpha_{b}=1
$$

Following equation is used to calculate physical parameters in the two-phase flow for a \& b:

$$
\left.\begin{array}{l}
\rho=\alpha \rho_{b}+(1-\alpha) \rho_{a} \\
\mu=\alpha \mu_{b}+(1-\alpha) \mu_{a}
\end{array}\right\}
$$

Where $\alpha$ is defined as,

$\alpha=1$, water

$\alpha=0$, air

To consider the effect of turbulence fluctuations, time-average of Navier-stokes equation should be taken, which is known as Reynolds-Averaged Navier-Stokes equation. The standard $k-\varepsilon$ turbulence model is used for turbulence modulation. 
$\mu_{t}=\rho C_{\mu} \frac{k^{2}}{\varepsilon}$

Where $k$ is turbulent kinetic energy, $\mu_{t}$ is turbulence viscosity, $C_{\mu}$ is constant of proportionality whose default value is 0.09 in fluent, $\varepsilon$ is the turbulence dissipation rate.

\section{Numerical implementation}

\subsection{Geometric Model}

The physical model used for present simulation consists of a 3-D partially filled water storage rectangular tank. Dimensions of the tank are $1.2 \mathrm{~m}$ in Length, $0.6 \mathrm{~m}$ in Height, and $1.2 \mathrm{~m}$ in Breadth. Two different cases of Water fill level in tank is used i.e. $60 \%$ and $80 \%$ of total height of tank and the rest part is occupied with air. Under the seismic excitation effect, tank experiences excessive sloshing effect that creates dynamic effect on tank wall. During computational simulation, to analyze the sloshing load pressure is monitored at a certain point on the right wall.

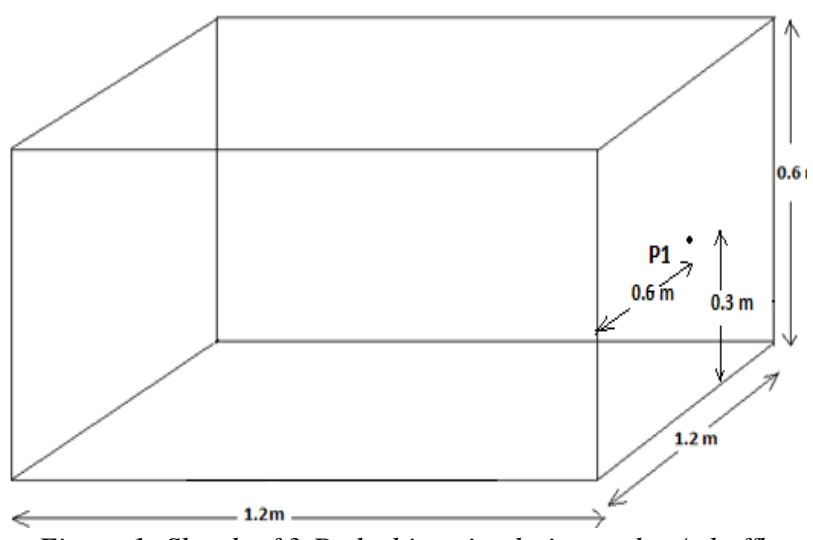

Figure 1: Sketch of 3-D sloshing simulation tank w/o baffle

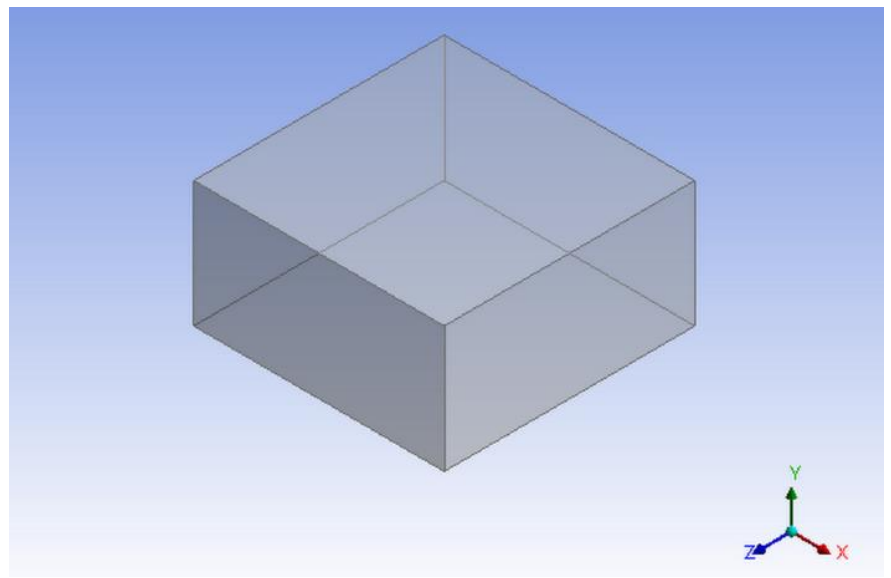

(a)

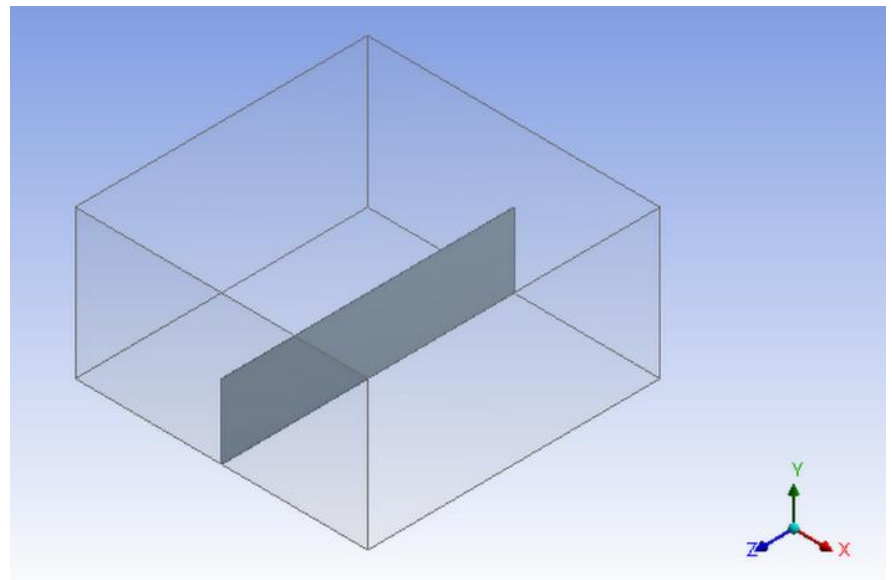

(b)

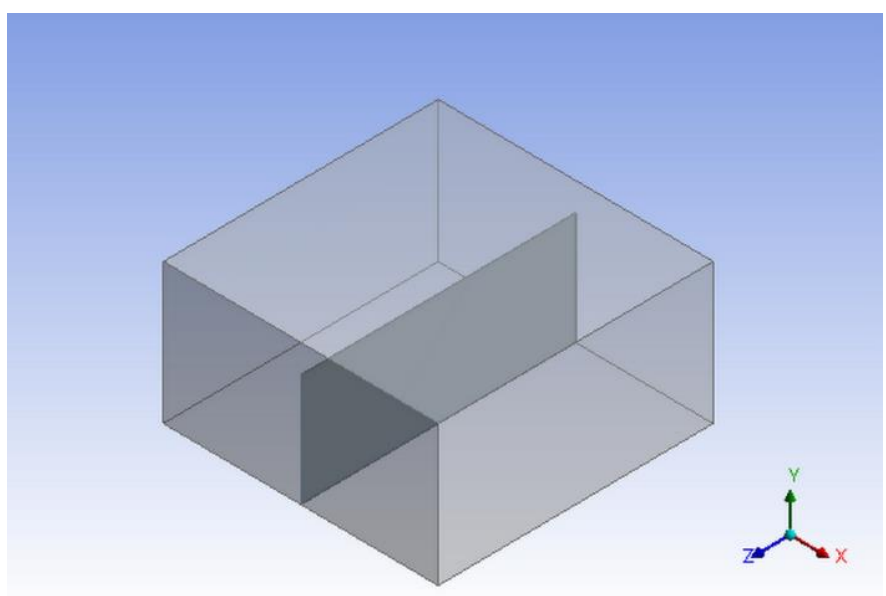

(c)

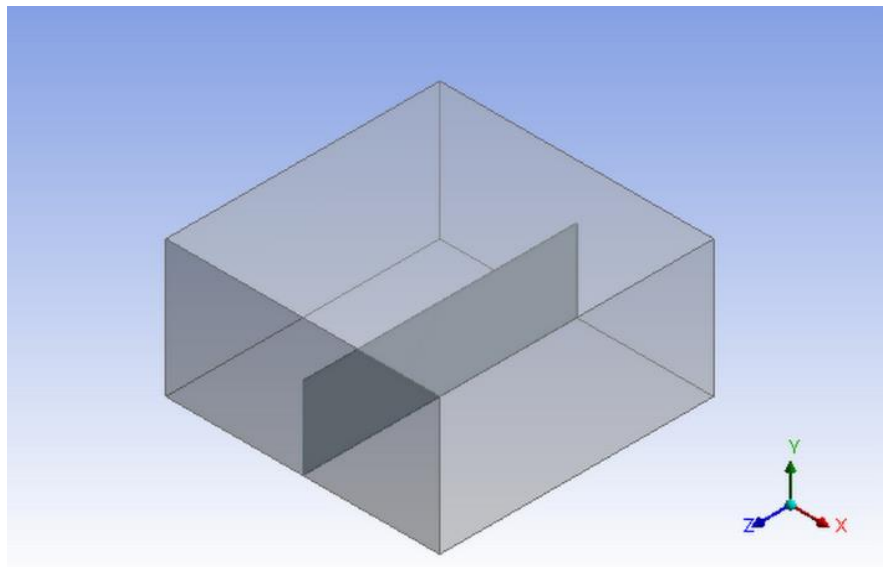

(d)

Figure 2: Rectangular tank with and without baffle (a) Tank without baffle (b) Tank with $0.3 \mathrm{~m}$ baffle, (c) Tank with $0.48 \mathrm{~m}$ baffle (d) Tank with $0.36 \mathrm{~m}$ baffle

To minimize the damage by sloshing on wall of tank, vertical baffle is arranged at the center of tank of thickness $2 \mathrm{~mm}$ and of varying heights that depends on level of water inside tank. 


\subsection{External excitations}

According to the linear theory of liquid sloshing, the modes of liquid sloshing corresponding to various immersion ratios can be obtained. The immersion ratio is defined as the ratio of the height to the length of the liquid level in a tank. For a 3-D rectangular tank, the lowest natural frequency of sloshing fluid in the tank can be computed by the following equation:

$$
\omega_{n}^{2}=\frac{n \pi g}{l} \tanh \left(\frac{n \pi h}{l}\right)
$$

Which represents the $\mathrm{n}^{\text {th }}$ mode oscillation frequency ' $\omega_{\mathrm{n}}$ ' in a container of length ' $l$ ' and fluid height ' $h$ '.

In this present work computational simulation with two different frequencies are performed. One at first order natural frequency and other at 0.93 times the natural frequency of sloshing.

The first order natural frequency and 0.93 times the natural frequency of sloshing for the liquid tank computed by above equation are:

$\omega_{\mathrm{n}}=4.34 \mathrm{rad} / \mathrm{s}$ (for $60 \%$ fill level)

$\omega_{\mathrm{n}}=4.67 \mathrm{rad} / \mathrm{s}$ (for $80 \%$ fill level)

$0.93 \omega_{\mathrm{n}}=4.03 \mathrm{rad} / \mathrm{s}$ (for $60 \%$ fill level)

$0.93 \omega_{\mathrm{n}}=4.34 \mathrm{rad} / \mathrm{s}$ (for $80 \%$ fill level)

The motion of the tank is purely translation and follows the sinusoidal base excitation. Motion of the tank can be represented by:

$$
\left.\begin{array}{l}
x_{T}=X \sin (\omega t) \\
\dot{x}_{T}=X \omega \cos (\omega t) \\
\ddot{x}_{T}=-X \omega^{2} \sin (\omega t)
\end{array}\right\}
$$

Where $x_{T}$ is displacement, $\dot{x}_{T}$ is horizontal translational velocity, $\ddot{x_{T}}$ is horizontal acceleration, $\mathrm{X}$ is the horizontal displacement amplitude, $\omega$ is the angular frequency and $t$ is time period of oscillation.

\subsection{Simulations Settings}

The commercial CFD code ANSYS-FLUENT is used for all the simulations presented in this work. The momentum and mass conservation equations are discretized using finite volume technique considering each cell as a control volume. The pressure-velocity equations are decoupled by the "Fractional Step" algorithm. The transport equation for the volume fraction is solved by the explicit time-marching scheme. The time step is 1e-5 s. The boundaries of the tank are set as non-slip walls, and the wave tank is initially static. Several user defined functions are plugged into the CFD code and work with the CFD cell zone conditions to implement the motions of the tank under the seismic external excitations.

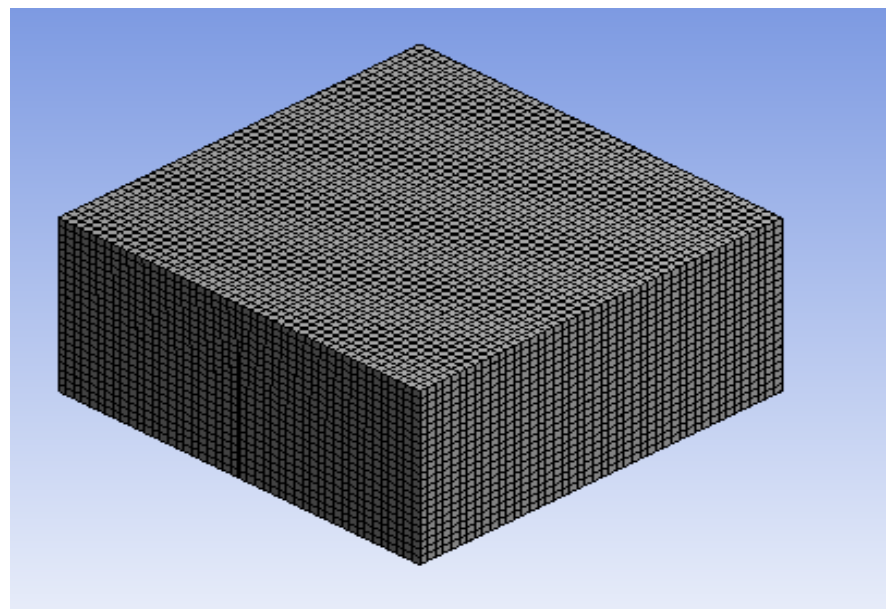

Figure 3: Computational grid of rectangular tank for water storage

\section{Results and discussions}

\subsection{Force variation without baffle}

Fig. 4 shows simulation results of comparison of forces between two different excitation frequencies $\left(\omega_{\mathrm{n}} \& 0.93 \omega_{\mathrm{n}}\right)$. Graph represents the variation of forces (Longitudinal and lateral), when the tank is not equipped with baffle and is $60 \%$ fill.

Fig. 5 shows simulation results of comparison of forces between two different excitation frequencies $\left(\omega_{\mathrm{n}} \& 0.93 \omega_{\mathrm{n}}\right)$. Graph represents the variation of forces (Longitudinal and lateral), when the tank is not equipped with baffle and is $80 \%$ fill.

When the tank is excited by resonance frequency, tank wall experiences higher magnitude of longitudinal forces and the deviation of amplitudes is much high. For lateral forces magnitude of forces is almost same but variation is greater at resonance condition. At $80 \%$ fill level, the magnitude of lateral force is higher as compared to $60 \%$ fill level since the impact on roof will be more at higher fill level.

For the case of the tank without equipped with baffle, liquid sloshing force is too high and can cause the damage of the side walls and impact the roof of the tank.

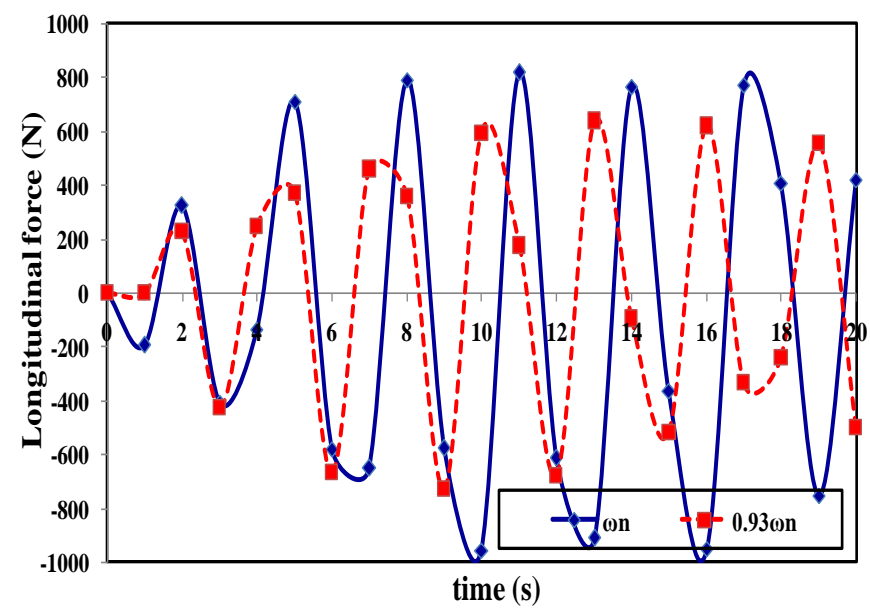

(a) 


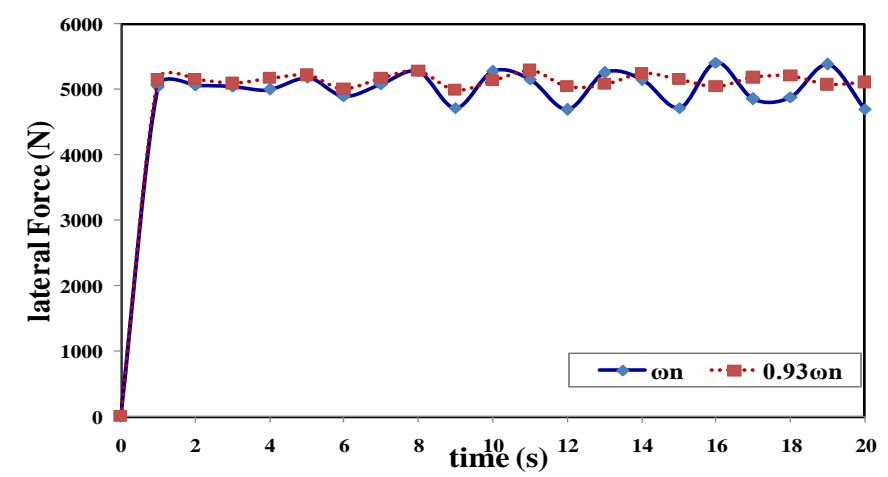

(b)

Figure 4: (a) Longitudinal forces variation at $\omega$ and $0.93 \omega n$ at $60 \%$ fill level, (b) Lateral forces variation at wn and $0.93 \omega n$ at $60 \%$ fill level

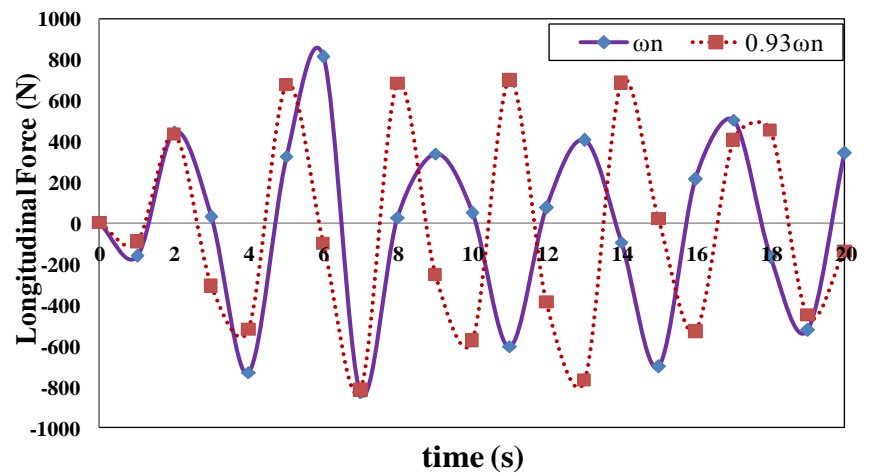

(a)

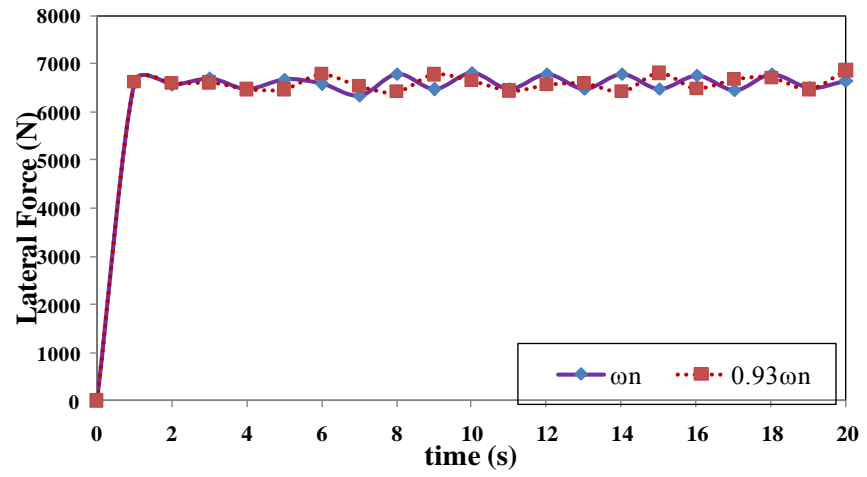

(b)

Figure 5. (a) Longitudinal forces variation at $\omega_{n}$ and $0.93 \omega_{n}$ at $80 \%$ fill level, (b) Lateral forces variation at $\omega_{n}$ and $0.93 \omega_{n}$ at $80 \%$ fill level

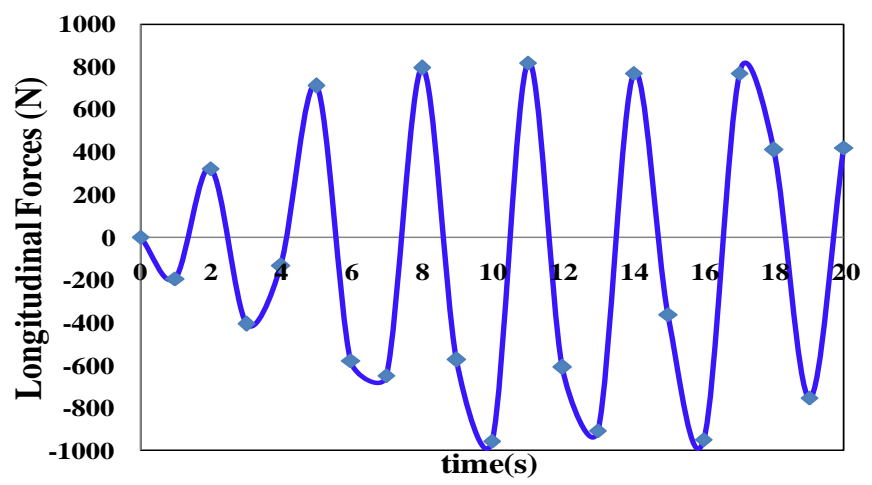

(a)

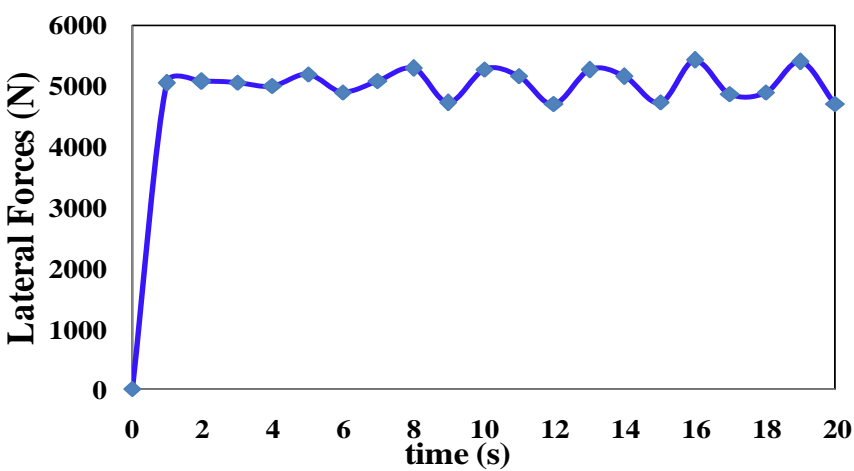

(b)

Figure 6: Variation of Forces (Longitudinal and Lateral) at $\omega_{n}$ at $60 \%$ fill level

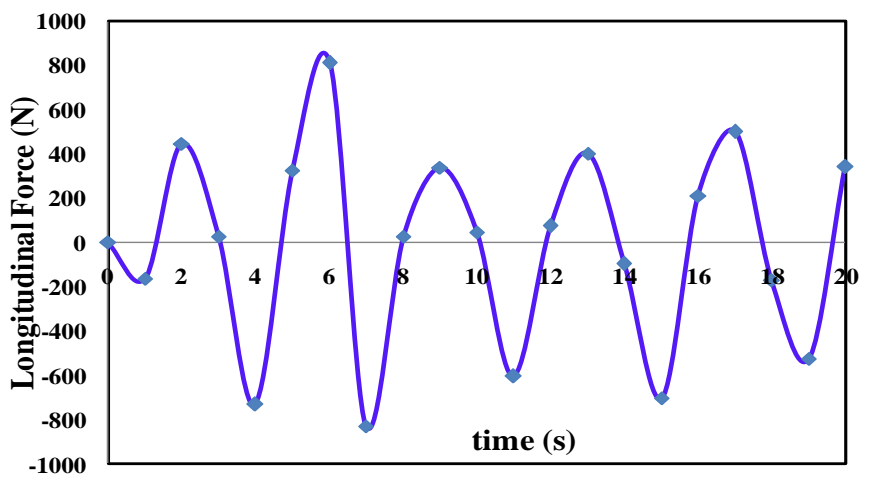

(a)

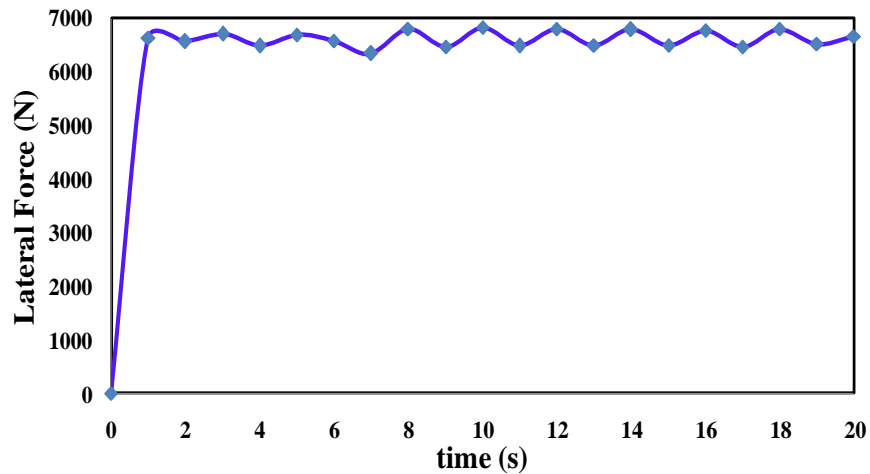

(b)

Figure 7: Variation of Forces (Longitudinal and Lateral) at $\omega_{n}$ at $80 \%$ fill level

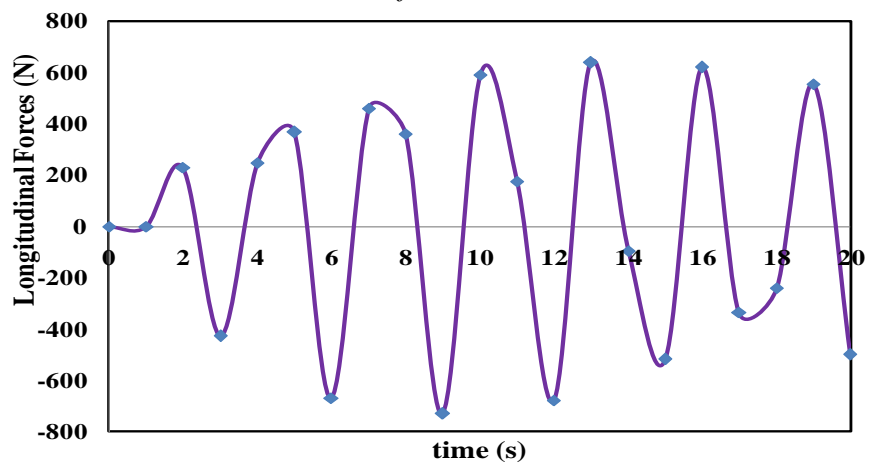

(a) 


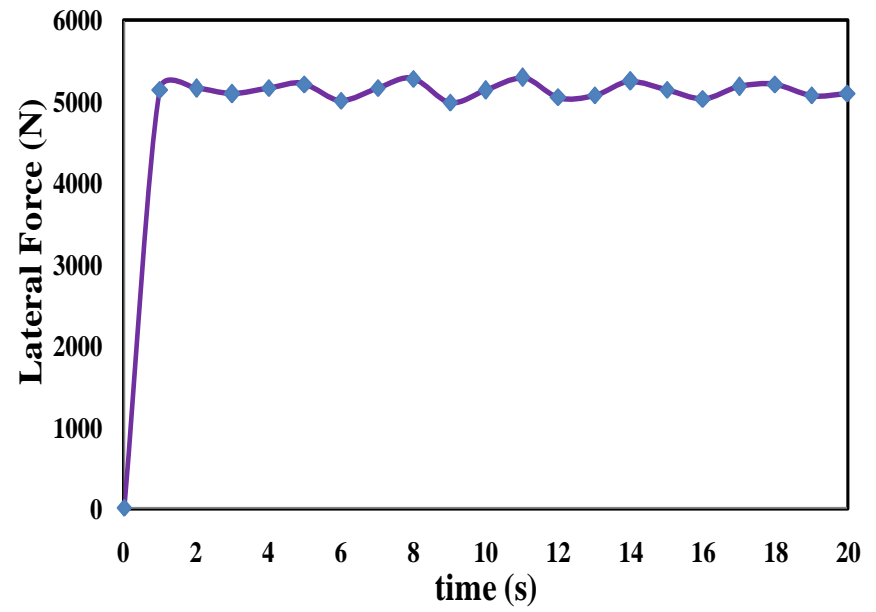

(b)

Figure 8: Variation of Forces (Longitudinal and Lateral) at $0.93 \omega_{n}$ at $60 \%$ fill level
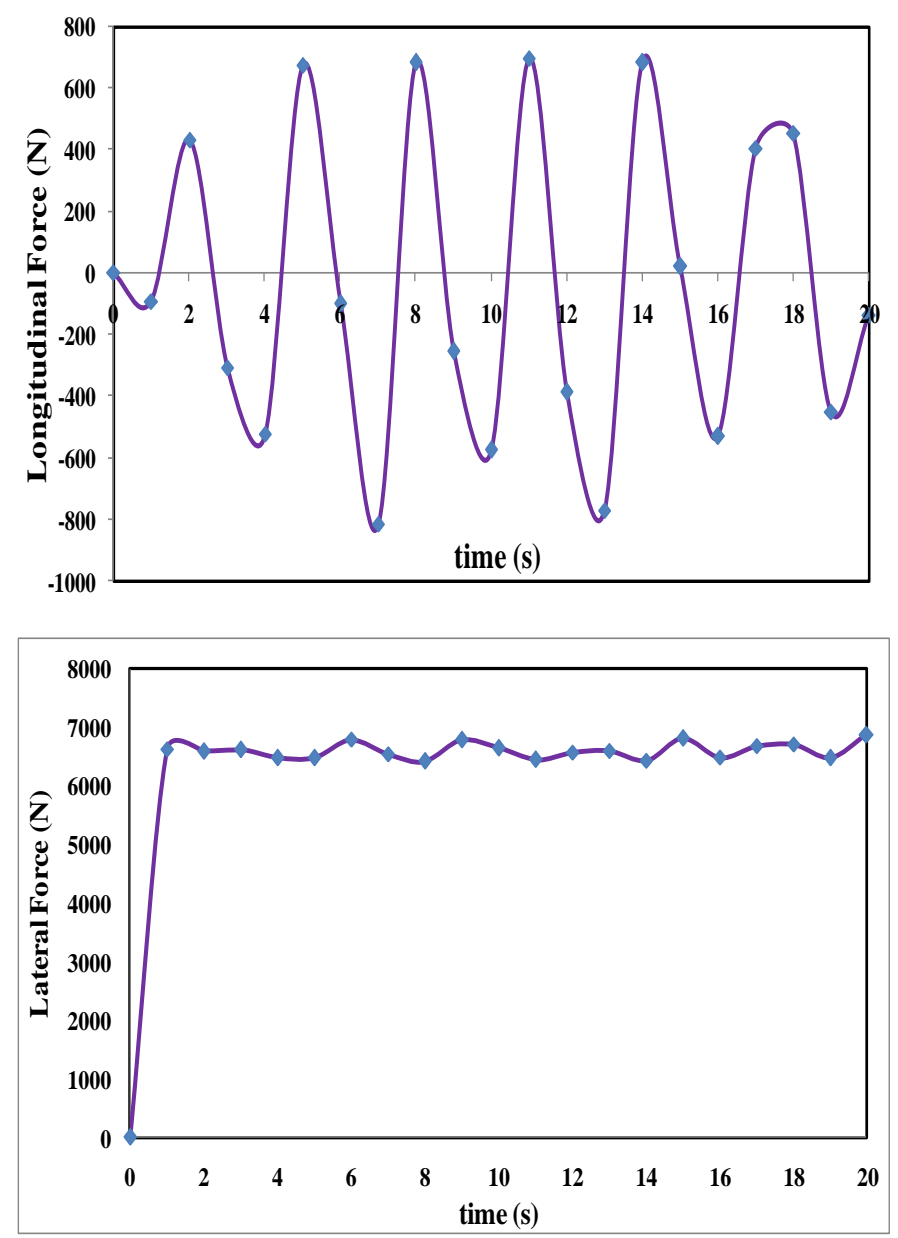

Figure 9: Variation of Forces (Longitudinal and Lateral) at 0.93wn at $80 \%$ fill level

Fig 8 \& Fig 9 represent the forces acting on side walls of the tank and the lateral forces acting on tank when the excitation frequency is 0.93 times of natural frequency of tank.

\subsection{Force variation with baffle height $0.3 \mathrm{~m}$}

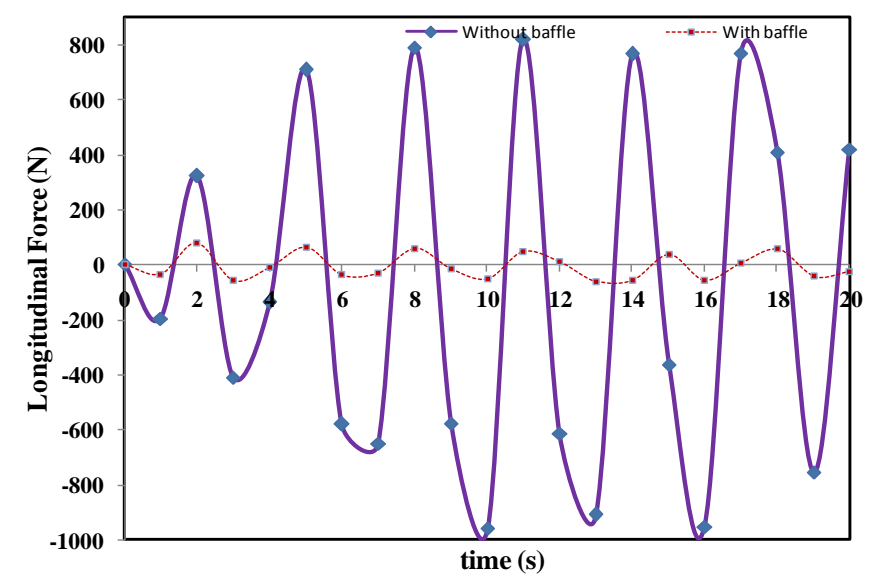

Figure 10 : Comparison of Longitudinal Force at $\omega_{n}$ for $60 \%$ fill with baffle of $0.3 m$ height and without baffle

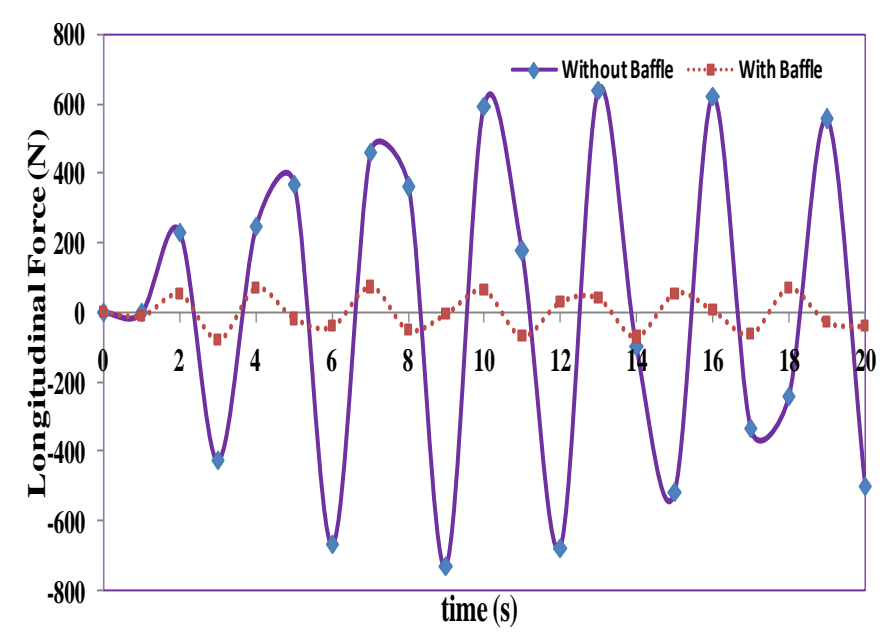

Figure 11: Comparison of Longitudinal Force at $0.93 \omega_{n}$ for $60 \%$ fill with baffle of $0.3 \mathrm{~m}$ height and without baffle.

When the vertical baffle is mounted on the tank Fig 10 \& Fig 11 , motion of the liquid becomes restricted due the obstruction created by baffle; free surface movement of the liquid becomes stable and reduces the magnitude of forces acting on side walls and roof.

\subsection{Force variation with different baffle heights $(0.3 \mathrm{~m}, 0.36 \mathrm{~m}$, $0.48 m$ )}

As the baffle height approaches to water fill level in tank Fig 12, Fig 13 \& Fig 14, free liquid surface shows almost linear behavior and hence the sloshing effect minimises and damage can be minimized. 


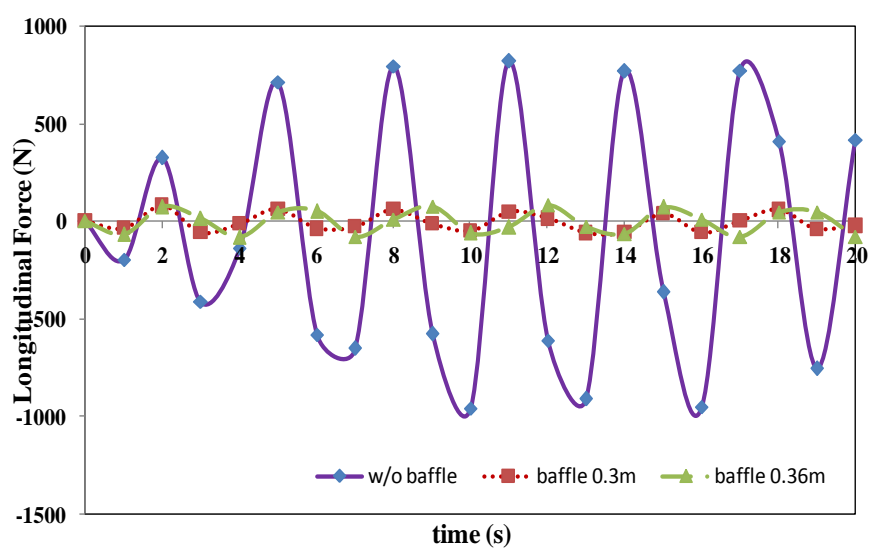

Figure 12: Longitudinal forces variation for $60 \%$ fill at $\omega_{n}$ with and without baffle

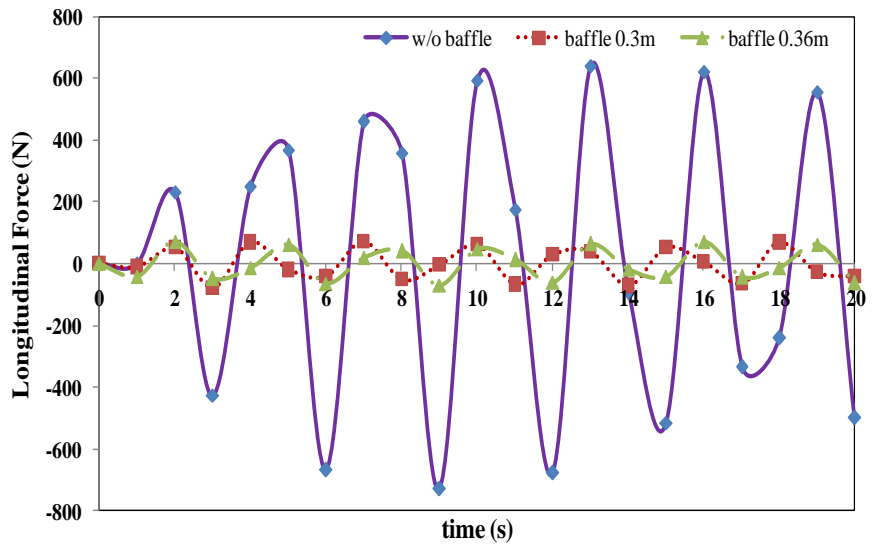

Figure 13: Longitudinal forces variation for $60 \%$ fill at $0.93 \omega_{n}$ with and without baffle

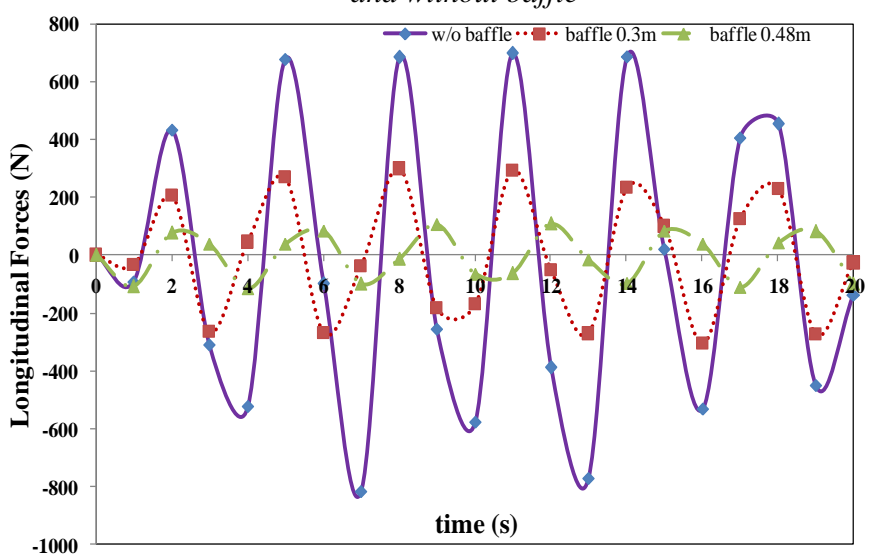

Figure 14: Longitudinal forces variation for $80 \%$ fill at $0.93 \omega_{n}$ with and without baffle

Fig 15. shows the pressure history at point P1 of the tank when tank is excited by natural frequency and is without baffled condition.

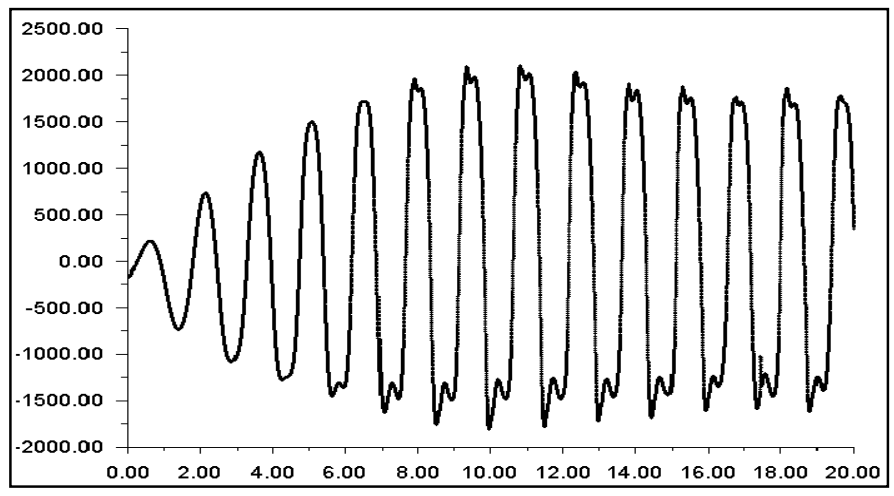

(a)

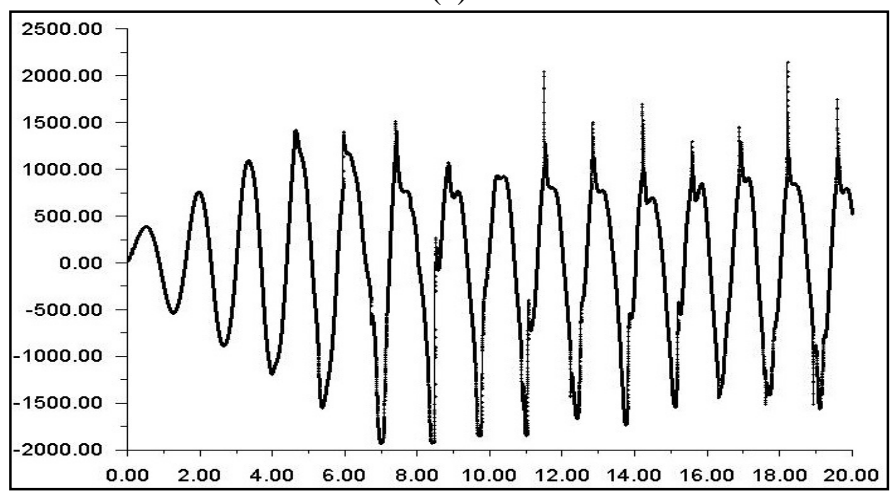

(b)

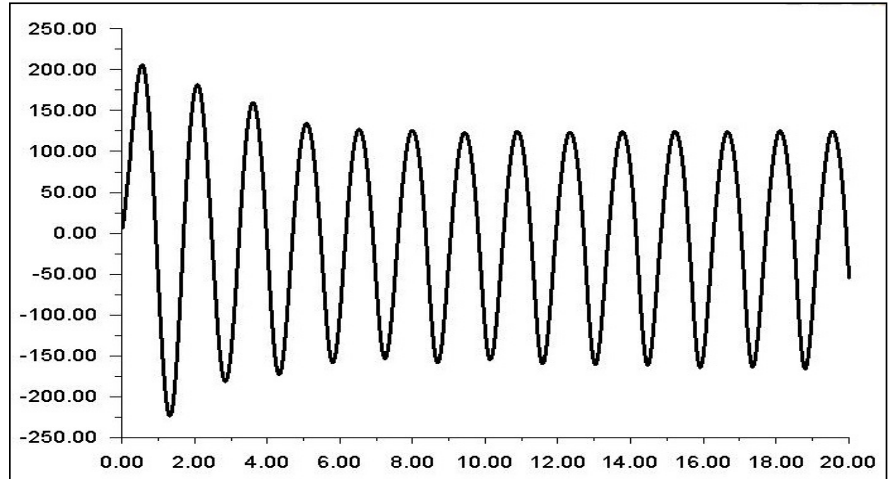

(c)

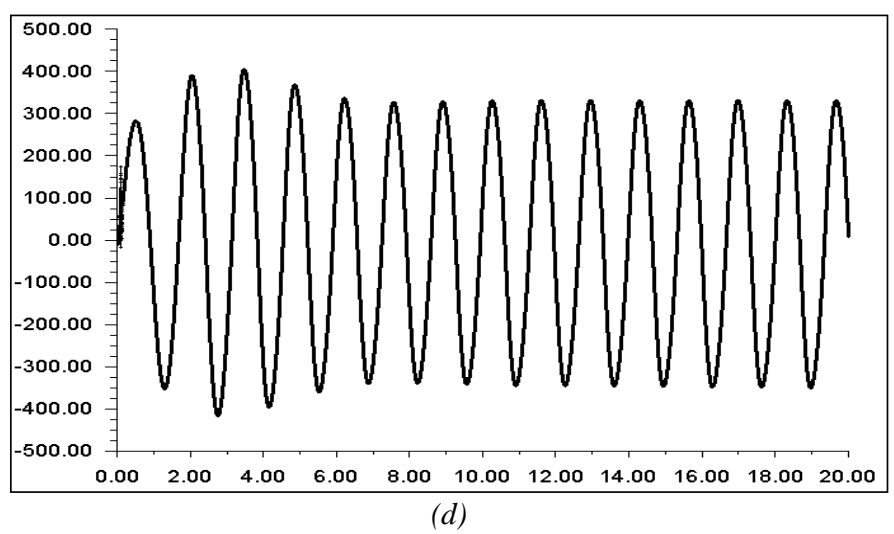

Figure 15: Static Pressure history at point P1

(a) $60 \%$ fill without baffle (b) $80 \%$ fill without baffle (c) $60 \%$ fill with $0.3 \mathrm{~m}$ baffle $(d) 80 \%$ fill with $0.3 \mathrm{~m}$ baffle 
When a tank is subjected to seismic excitation, waves are produced in the free liquid surface and thus the liquid exerts a pressure on the tank walls. This pressure variation with time at point P1 is represented in Fig .15(a) \& (b).If baffle of $0.3 \mathrm{~m}$ height is placed inside tank, the pressure intensity reduces by a marginal level and thus reduces the damage to the tank. Fig 15 (c) \& (d) represents pressure variation when the tank is equipped with baffle and tank is filled with water at $60 \%$ level and $80 \%$ level.

\section{Case (a): for $80 \%$ fill}

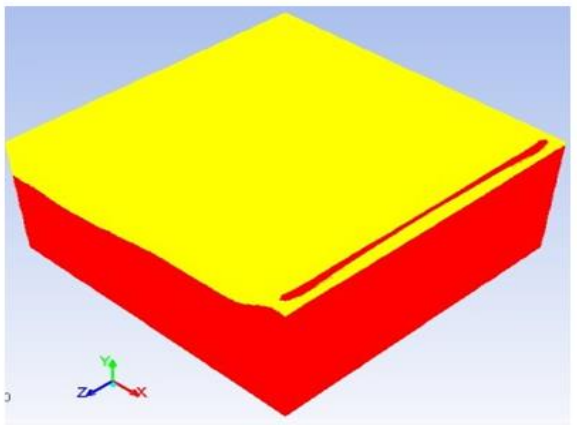

$\mathrm{t}=5 \mathrm{sec}$

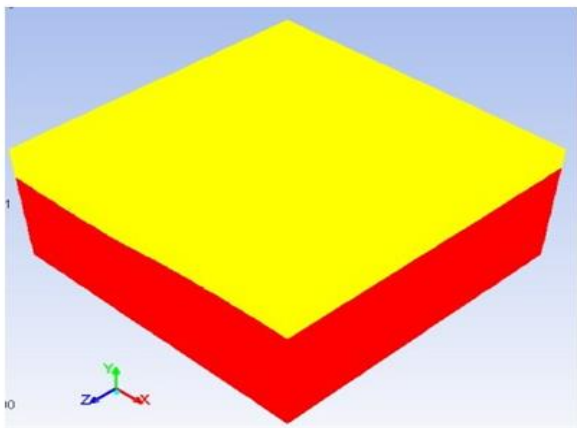

$\mathrm{t}=5 \mathrm{sec}$

Case (b): for $60 \%$ fill

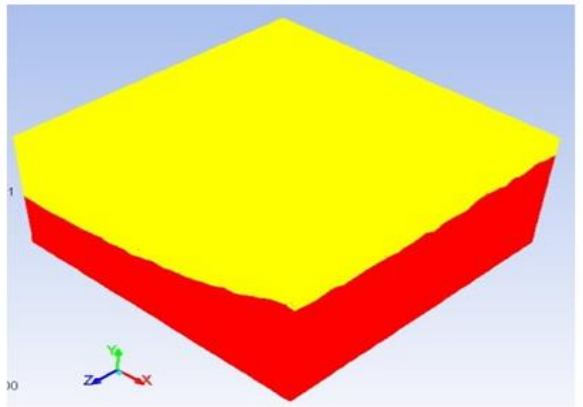

$\mathrm{t}=5 \mathrm{sec}$

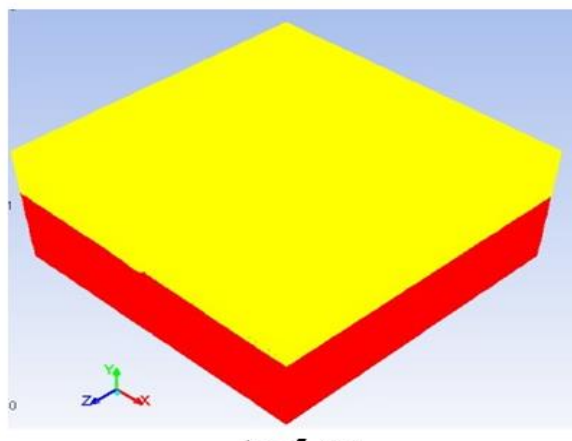

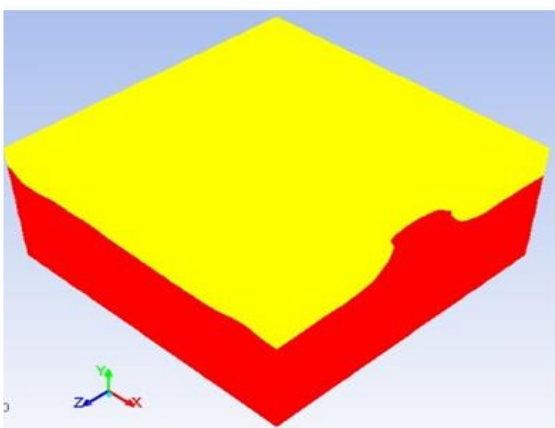

$\mathrm{t}=10 \mathrm{sec}$
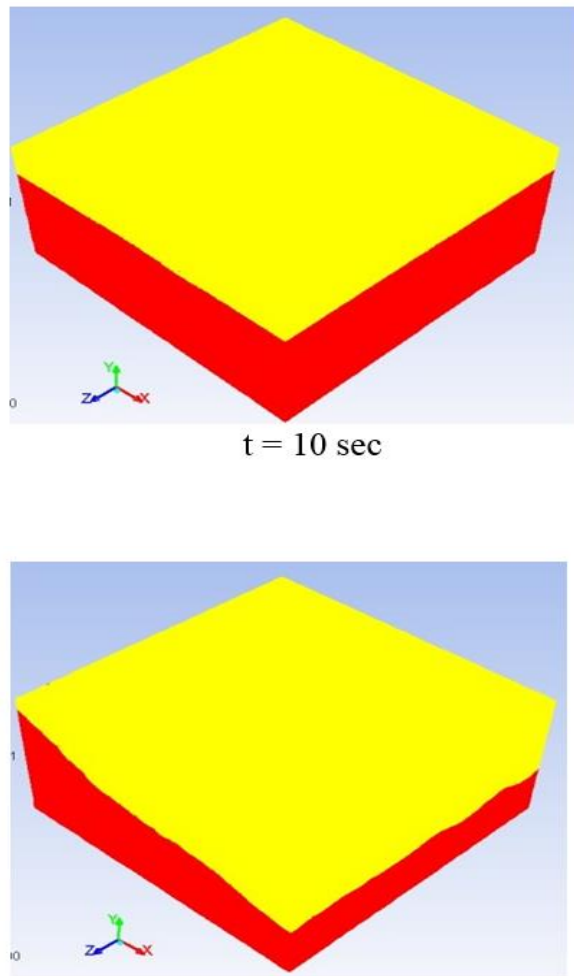

$\mathrm{t}=10 \mathrm{sec}$

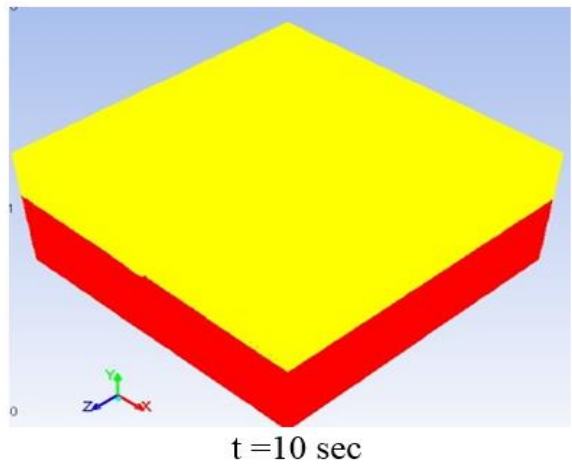

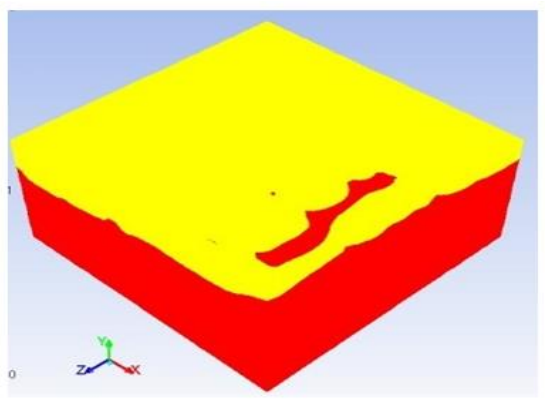

$\mathrm{t}=20 \mathrm{sec}$
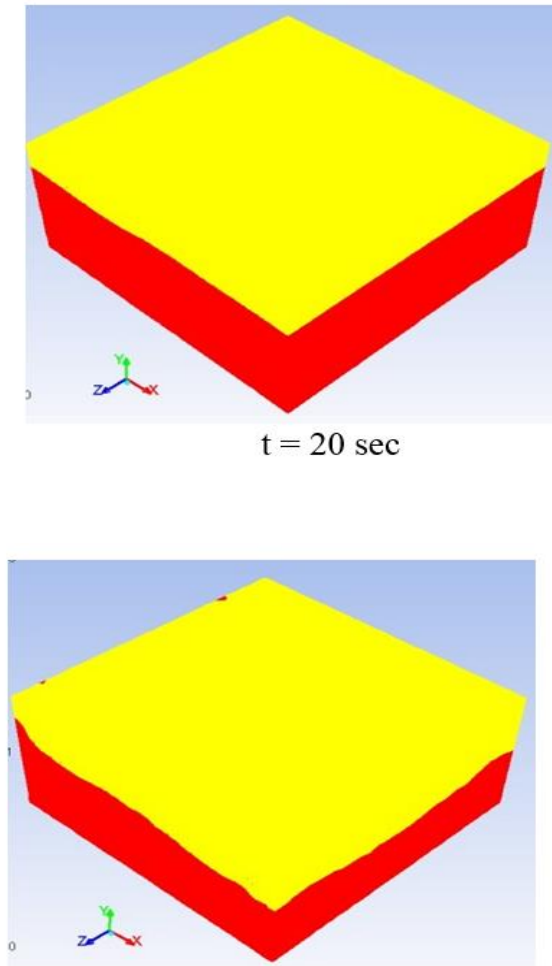

$\mathrm{t}=15 \mathrm{sec}$

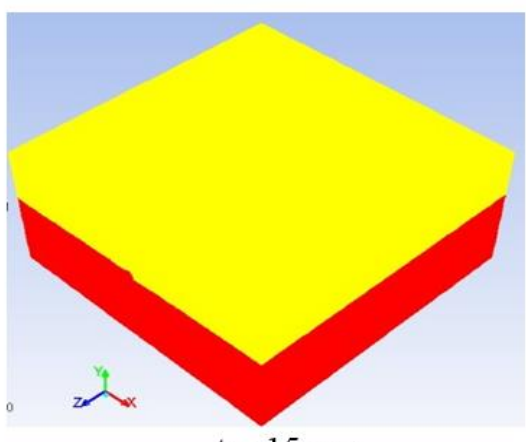

Figure 16: Representation of Free Surface under Seismic Excitation 
Following Fig. 16 represent the position of free surface under seismic excitation when the tank is excited by first order natural frequency for $80 \%$ and $60 \%$ fill level with and without vertical baffle of height equal to fill level.

\section{Conclusions}

Sloshing phenomenon in 3-D water storage rectangular tank is investigated. The extremity of liquid sloshing and its pressure loads depends on the tank configuration, liquid fill level, amplitude and nature of the tank excitement. It was clear from the simulations done computationally, at different time steps, that sloshing can effectively reduce dynamic stability of container. The forces acting on walls increases as the excitation frequency reaches near to resonance frequency of the tank.

For the further research, different designs of baffles can be examined to reduce the tank sloshing phenomenon. Baffles are devices that are employed in tanks to reduce the effect of slosh dynamics. The proportions of the baffles can also be analyzed for further decline in the sloshing magnitude. Sloshing under different seismic excitations amplitude can also be investigated.

\section{References}

[1] Ibrahim RA (2005). Liquid sloshing dynamics: theory and applications. Cambridge University Press, Cambridge

[2] Biswal K.C., Bhattacharya S.K., Sinha P.K.(2003). "Free-Vibration Analysis Of Liquid-Filled Tank With Baffles. Journal of Sound and vibration" 259(1), 177-192

[3] Akyildiz H., Unal.E. (2005), "Experimental investigation of pressure distribution on a rectangular tank due to the liquid sloshing" Ocean Engineering. 32:1503-1516

[4] T. Krit, C. Suvanjumrat, "The Effect of Baffles on Fluid Sloshing inside the Moving Rectangular Tank", Journal of Research and Applications in Mechanical Engineering. Vol. 1 No.2

[5] Lin P (2008). "Numerical modeling of water waves". Taylor \& Francis, London.

[6] Faltinsen OM (1978). "A numerical nonlinear method of sloshing in tanks with two-dimensional flow". Journal of Ship Research, 22(3), 193-202.

[7] Hill DF (2003). "Transient and steady-state amplitudes of forced waves in rectangular basins". Physics of Fluids, 15(6), 1576-1587.

[8] Ling Hou, Fangcheng Li, Chunliang Wu (2012), "A Numerical Study of Liquid Sloshing in a Two-dimensional Tank under External Excitations", J. Marine Sci. Appl. Vol 11: 305-310

[9] J.H. Jung, H.S.Yoon, C.Y.Lee, S.C.Shin (2012), "Effect of the vertical baffle height on the liquid sloshing in a three-dimensional rectangular tank", Ocean Engineering. Vol 44: 79-89

[10] V. Singal, Jash Bajaj, Nimish Awalgaonkar, Sarthak Tibdewal (2014), "CFD Analysis of a Kerosene Fuel Tank to Reduce Liquid Sloshing", Procedia Engineering. Vol 69: 1365 - 1371

[11] T. Kandasamy, S. Rakheja, A.K.W. Ahmed, (2010), "An Analysis of Baffles Designs for Limiting Fluid Slosh in Partly Filled Tank Trucks", The Open Transportation Journal. Vol 4: 23-32

[12] K. J. Craig - T. C. Kingsley (2007), "Design optimization of containers for sloshing and impact", DOI 10.1007/s00158-006-0038-6. Vol 33: 71-87

[13] Godderidge B., Stephen Turnock, Mingyi Tan, Chris Earl (2009), "An investigation of multiphase CFD modelling of a lateral sloshing tank", Computers \& Fluids. Vol 38: 183-193

[14] M. Eswaran, U.K. Saha, D. Maity (2009), "Effect of baffles on a partially filled cubic tank: Numerical simulation and experimental validation", Computers and Structures. Vol 87: 198-205

[15] L. Khezzar, A. C. Seibi, A. Goharzadeh , (2009), "Water Sloshing in Rectangular Tanks - An Experimental Investigation \& Numerical Simulation", International Journal of Engineering (IJE), Vol 3: Issue (2) 174-184

[16] P.K.Panigrahy, U.K.Saha, D.Maity, (2009), "Experimental studies on sloshing behavior due to horizontal movement of liquids in baffled tanks", Ocean Engineering. Vol 36:213-222

[17] Choun, Y.-S., Yun, C.-B., (1996). Sloshing characteristics in rectangular tanks with a submerged block. Comput. Struct. 61 (3), 401-413.

[18] Choun, Y.-S., Yun, C.-B., (1999). Sloshing analysis of rectangular tanks with a submerged structure using small-amplitude wave theory. Earthquake Eng. Struct. Dyn. 28 (7), 763-783.

[19] K.M. Kleefsman, G. Fekken, A. E. Veldman, B. Iwanowski, and B. Buchner, , (2005) "A volume-of-fluid based simulation method for wave impact problems," Journal of Computational Physics, vol.206, no. 1, pp. 363-393.

[20] R. A. Ibrahim, V. N. Pilipchuk, and T. Ikeda, (2001) "Recent advances in liquid sloshing dynamics," Applied Mechanics Reviews, vol. 54, no. 2, pp. 133-199

[21] O. M. Faltinsen and A. N. Timokha, (2009) Sloshing, Cambridge University Press, Cambridge, UK

[22] B.-F. Chen and R. Nokes, (2005)"Time-independent finite differencevanalysis of fully non-linear and viscous fluid sloshing in a rectangular tank," Journal of Computational Physics, vol. 209, no.1, pp. 47-81.

[23] B.-F. Chen, (2005) "Viscous fluid in tank under coupled surge, heave, and pitch motions," Journal of Waterway, Port, Coastal and Ocean Engineering, vol. 131, no. 5, pp. 239-256,.

[24] G. X. Wu, Q. W. Ma, and R. Eatock Taylor, (1998) "Numerical simulation of sloshing waves in a 3D tank based on a finite element method," Applied Ocean Research, vol. 20, no. 6, pp. 337-355

[25] S. Mitra, P. P. Upadhyay, and K. P. Sinhamahapatra, (2008). "Slosh dynamics of inviscid fluids in two-dimensional tanks of various geometry using finite element method," International Journal for Numerical Methods in Fluids, vol. 56, no. 9, pp. 1625-1651.

[26] Akyildiz, H ,Unal, E.,(2005).Experimental investigation of pressure distribution on a rectangular tank due to the liquid sloshing. Ocean Engineering 32(11-12), 1503-1516.

[27] Cho, J.R., Lee, H.W., (2004). Numerical study on liquid sloshing in baffled tank by nonlinear finite element method. Computer Methods in Applied Mechanics and Engineering 193 (23-26), 2581-2598.

[28] Cho, J.R., Lee, H.W., Ha, S.Y., (2005). Finite element analysis of resonant sloshing response in a 2D baffled tank. Journal of Sound and Vibration 228 (4-5), 829-845.

[29] Graham, E.W. and Rodriguez, A.M. (1952). "Characteristics of fuel motion which affect airplane dynamics", Journal of Applied Mechanics, 19, 381-388.

[30] Housner, G.W. (1954), "Effect of foundation compliance on earthquake stresses in multi-story buildings", Bull.Seism. Soc. Am., 44, pp551-569.

[31] Jin, H., Liu, Y., Li, H.-J., (2014). Experimental study on sloshing in a tank with an inner horizontal perforated plate. Ocean Eng. 82, 75-84.

[32] Jin Heng, Liu Yong, Li Huajun, and Fu Qiang, (2017), Numerical Analysis of the Flow Field in a Sloshing Tank with a Horizontal Perforated Plate, J. Ocean Univ. China (Oceanic and Coastal Sea Research), 16 (4): 575-584

[33] P K Nema, (2014) Computational Study of Sloshing Behavior in 3-D Rectangular Tank with and Without Baffle Under Seismic Excitation. Master Thesis, National institute of technology, Rourkela.

Cite this article as: Puneet Kumar Nema, Kapil, Computational analysis of sloshing in 3-D rectangular tank under seismic excitation with and without baffle, , International Journal of Research in Engineering and Innovation Vol-3, Issue-5 (2019), 330-338. 